\title{
Maternal Participation Program for Enhancing Growth and Neurobehavioral Development of Very and Moderately Preterm Infants: A Case Study
}

\author{
Nethong NAMPROM*, Wilawan PICHEANSATHIAN, \\ Usanee JINTRAWET and Jutamas CHOTIBANG
}

\author{
Faculty of Nursing, Chiang Mai University, Chiang Mai 50210, Thailand
}

('Corresponding author’s e-mail: nethong.namprom@cmu.ac.th)

Received: 3 April 2017, Revised: 7 October 2017, Accepted: 16 November 2017

\begin{abstract}
A Maternal Participation Program in the context of Thai culture was developed based on the Neonatal Integrative Developmental Care model. A multiple case study design was used to explore the feasibility and acceptability and potential outcomes of implementing the program in a Thai NICU. The potential outcomes were growth and neurobehavioral development of very and moderately preterm infants. This program consisted of education and monitoring strategies including 4 teaching and practice sessions of 6 care practices, which included optimizing nutrition, positioning and handling, safeguarding sleep, promoting a healing environment, minimizing stress, and protecting the skin. The maternal participation program with education strategies was implemented one week after the infant's admission to an NICU. Strategies to monitor the maternal participation level and to boost maternal participation were used. The maternal participation program was beneficial for 3 Thai mothers. They gave positive feedback regarding the feasibility and acceptability of the program, as well as the readability of the "My Baby" handbook. The level of maternal participation in caring for preterm infants increased after being involved in this program. Additionally, preterm infants' body weight and neurobehavioral scores dramatically increased. Further studies of this program need to be conducted in a randomized controlled trial design.
\end{abstract}

Keywords: Neonatal, maternal participation, growth, neurobehavioral development, preterm infants

\section{Introduction}

Growth and development in the early life of premature infants is very crucial because at that stage they are at risk of health problems that may affect their development in later life. Recently, it was found in the United States [1], as well as in Thailand [2], that the survival of preterm infants increases with gestational and postnatal age. This may be the result of advanced medical practice and nursing care leading to the infants' survival outside of the uterine environment. However, preterm infants still experience health problems, especially severe respiratory disorders which require treatment in an NICU [3].

Being hospitalized in an NICU, preterm infants are put in a different environment from the maternal womb and encounter noxious stimuli. These put them at risk of health problems such as cerebral intraventricular hemorrhage, sepsis, gastrointestinal infections, retinopathy of prematurity, and lung damage [4], and delayed growth and development [5]. A study showed that preterm infants with an illness had a lower neurobehavioral score compared to healthy term infants [6]. It was also found that preterm infants had a prolonged length of hospital stay and more days of ventilation usage than infants with an older gestational age [7]. Furthermore, during that time, because the mothers of these infants had already been discharged, infants suffered from maternal and infant separation. 
http://wjst.wu.ac.th

Hospitalized preterm infants in an NICU are separated from their mothers, particularly among very preterm and moderately preterm infants. Mothers can only take a look at their infants and have fewer opportunities for contact with their infants [8]. Most mothers whose preterm infants were in an NICU experienced feelings of incomprehension [9], lack of control [10], powerlessness [11], and inability to care for their infants [12,13]. These mothers also mentioned feeling overwhelmed by the environment of the NICU which included equipment, noise, and light. Furthermore, they felt that they had to struggle to get connected with their infant because of the lack of support from the healthcare provider [14]. Many mothers felt a lack of ownership and had a sense of alienation towards their infant and the unit [10]. All of this interrupted and restricted their normal parenting [11]. Two studies confirmed that stress on neonates was a consequence of maternal deprivation which in turn affected their growth and development $[15,16]$. It was found that maternal participation in providing care for the preterm infant was shown to promote and facilitate growth [17], the maturation and connectivity of white matter [18] and neurofunctional development [19]. Thus, maternal participation in caring for preterm infants should be encouraged.

Four systematic reviews on the early interventions involving parents for preterm infants indicated that 3 key components for improving mother and preterm outcomes should be included psychosocial support, educational support, and therapeutic developmental interventions targeting the infants [20-23]. The Neonatal Integrative Developmental Care (IDC) model proposed by Altimier and Phillips [24] provides neuroprotective intervention strategies which contain those 3 key components as mentioned above. The model provides practical guidance for mothers on 'how to' deliver developmental care to their preterm infants in an NICU, which is called partnering. To achieve the goal of partnership this model must include 6 dimensions caring for preterm infants as follows: promoting a healing environment, positioning and handling, safeguarding sleep, minimizing stress and pain, protecting the skin, and optimizing nutrition [24]. However, previous studies did not include all components. It is anticipated that a comprehensive intervention program containing 3 key components aims at improving maternal participation, growth, and development of preterm infants have to be developed and tested.

In Thailand, the study by Pongjaturavit and Harrigan [25] indicated that Thai parents need more assistance than Western parents to enhance their relationship and communication with nurses. They also need more information about the care of their hospitalized child. Moreover, Thai parents provide care for their hospitalized child in different ways from Western parents. Studies in Thailand found that most mothers participate in infant care in the NICU, but they need to perform more activities than they do currently [26-28]. These activities include routine care, technical care, information sharing, and decision making. An interesting finding about mother participation in a Thai NICU relates to the "kreng jai attitude" which in Thai culture means "feeling consideration for another person, not wanting to impose or cause another person trouble, or hurt his/her feelings" [29]. It is the nature of Thai people that limited mothers from caring for their babies. This finding was similar to the study by Chaudhuri, Easterbrooks, \& Davis [30] which found that cultural background predicted parenting style. Health care personnel should be aware of the issue of culturally sensitive care practices in an NICU in order to facilitate and support the mothers of preterm infants [31]. Thus, it is necessary to identify Thai cultural underpinnings of parent participation in the care of a hospitalized infant in order to promote maternal participation in caring for preterm infants.

This article presents the Maternal Participation Program in the Thai context. The central core of the program is nurse's partnering with a mother to perform 6 care practices as mentioned in the IDC model to promote maternal participation in caring for preterm infants in order to enhance their growth and neurobehavioral development. The aim of the study was to test the program with 3 Thai mothers of very preterm and moderately preterm infants using a case study approach with the hope of refining the process and establishing a protocol to study larger samples, and to determine the feasibility and acceptability of the program. 
http://wjst.wu.ac.th

\section{Materials and methods}

A 3-case study design provided an in-depth investigation of phenomena in a real-life context using both quantitative and qualitative data collection strategies to reveal the perception of mothers of preterm infants regarding the feasibility and acceptability of the Maternal Participation Program. In addition, the readability of the handbook in the program was also measured. Mothers of preterm infants were approached on admission and screened for their eligibility including 1) age of 18 years or older, 2) ability to read and speak Thai, 3) having given birth to a preterm infant with a gestational age of between 28 weeks to 32 weeks as assessed by the New Ballard score, and 4) being a mother of a preterm infant who was a singleton birth born at the study site and hospitalized in the NICU for the first time, with an anticipated survival, no congenital abnormality or disease, and a birth weight of less than 2,500 grams. The mothers were asked to sign an informed consent form prior to participation. The protocol and consent forms were approved by the research ethics committee of the Faculty of Nursing, and the Faculty of Medicine, Chiang Mai University.

\section{Procedures}

The study was divided into 3 phases. The first phase was to collect the demographic data of the mother-preterm infant dyads in the NICU. The second phase was the implementation of the Maternal Participation Program within one week after admission of the preterm infant to the NICU. The central core of the program was partnering between nurse and mother by providing education and improvement strategies. The researcher aimed to develop this program for nurses to educate and monitor preterm infants' mothers on delivering 6 care practices to their infants. Education strategies included teaching and practice sessions. Four teaching sessions were provided for the mothers. These sessions consisted of four $1 \mathrm{~h}$ lecture classes on 6 topics of maternal participation in the practice of infant care: providing a healing environment, positioning and handling, safeguarding sleep, minimizing stress and pain, protecting the skin, and optimizing nutrition. Four, $1 \mathrm{~h}$ teaching sessions consisted of a 5 min introduction, a 40 min multimedia presentation in conjunction with the nurse's explanation, a 10 min mother sharing opportunity, and a 5 min debriefing. These sessions enhanced the mothers' understanding by using media, including an education plan, multi-touch interactive multimedia, and the "My Baby" handbook. Practices of care were performed on an individual basis in a separate room and bedside care with the strategy of demonstration and return-demonstration. Practice sessions consisted of four $1 \mathrm{~h}$ practices regarding the activities during maternal participation in infant care following the teaching topics. Teaching media consisted of an education plan, multi-touch interactive multimedia, a handbook, and material for practicing (e.g., a doll, blankets, baby oil, etc.). The content of the MP Program was validated and approved by 5 experts.

The education component consisted of the following sessions:

Session 1: An initial session was conducted on days 1 - 2 after the preterm infant admission. The mother was invited by the researcher to the bedside of the infant and participated in infant care by closely looking at her hospitalized preterm infant. The mother was also provided with information about the NICU environment, her infant's illness, characteristics of preterm infants, and how to know your infant. The aim of this session was to build the awareness of the mother as an important person to her preterm infant.

Session 2: The session on optimizing nutrition and providing a healing environment was conducted on day 3. The mother was taught care practices including breastfeeding, intermittent kangaroo mother care, promoting sucking, effect of breast milk odor, colostrum mouth care, using a soft voice, and eye to eye contact with demonstrations and return-demonstrations. A handbook was given to the mother for review and as a guide to participation in taking care of her preterm infant.

Session 3: Teaching regarding the safeguarding of sleep and positioning and handling was conducted on day 5. The researcher trained the mother to recognize her infant's sleep state, and then the mother practiced positioning, along with facilitated tucking and handling through demonstration and return-demonstration in a private room at the bedside of the infant. 
http://wjst.wu.ac.th

Session 4: The session on minimizing stress and pain and protecting the skin was conducted on day 7. The mother was taught how to recognize cues from her infant, and then trained in facilitated tucking and light touching, as well as developmentally appropriate infant massage and care.

Day 4, 6, and 8 were given as the free day for mother to review activities of each care practice they had learned, as well as to break content. The whole intervention period, the researcher promoted maternal participation in 6 care practice using the improvement strategies.

Improvement strategies. Assistance, facilitation, and encouragement were used as improvement strategies to increase the level of maternal participation. The maternal participation level was monitored by using the Maternal Participation in Caring for a Preterm Infant's Checklist at day 14 and day 21 after program implementation. In case that the overall of mothers' participation level was less than $95 \%$, the researcher and participants discussed to find out the barriers and the solution. In the meantime the researcher kept providing assistant facilitation and encouragement for mother to participate in performing all activities of care practices. The researcher assisted mothers by staying close to the mother and helping her when she was unsure about performing an activity. The researcher encouraged the mother to practice daily care for her preterm infant if she was hesitant to perform activities when she came to visit. The mother was asked to notify the NICU's head nurse of her intention to participate in the care of her preterm infant. In addition, the researcher observed the behavior of the mother and gave consolation when she appeared stressed or anxious about the unstable condition of her preterm infant. The researcher provided information regarding treatment and help as requested, as well as using positive feedback to reassure the mother. Telephone contact and chat support via the Line application of social media were also used to exchange information and encourage and empower mothers by reducing their stress.

In the last phase, the preterm infant's body weight and neurobehavioral development were assessed to obtain the outcomes at day 14 and day 28 because the previous studies demonstrated that there was a difference in weight gain rate in the first two weeks and the fourth weeks of life [32,33] The infants' body weight was assessed by using a digital scale, and weight was measured in grams, whereas neurobehavioral development of the preterm infants was assessed by using The Neonatal Neurobehavioral Examination [34].

\section{Measurement and instruments}

The aim of measurement was to gauge the feasibility and acceptability of the program and the readability of the handbook. Maternal participation levels and the outcomes of preterm infants were also assessed.

\section{Measurement of the program}

Feasibility is defined as "the extent to which a new treatment, or an innovation, can be successfully used or carried out within a given agency or setting" [35]. It was assessed at each session of the intervention. Feasibility of the program implementation was based on whether mothers would participate in specific program activities, and assessed by monitoring retention of participants throughout the intervention period and daily recording of the infant's weight in the handbook.

Acceptability is defined as "the perception among implementation stakeholders that a given treatment, service, practice, or innovation is agreeable, palatable, or satisfactory" [35]. The mothers' perspectives and experiences in the intervention, including comfort level and their perceptions of the program content, components, duration, and intensity were considered as acceptability of stakeholder. Acceptability was evaluated after completion of the intervention using both qualitative and quantitative data. Qualitative data were collected using a semi-structured interview and a self-report questionnaire at the end of the program. The participants were interviewed regarding their opinion of the content, strategy, method, and duration of the program. A self-report questionnaire asked about the mother's concerns regarding the program and any uncomfortable feelings she had. The questionnaire consisted of 7 items, including the helpfulness of the overall program, the mother's comfort with using multi-touch interactive multimedia, satisfaction of using multimedia, satisfaction with the practice sessions, satisfaction with the monitoring strategies, appropriateness of the program for the mother of a preterm infant, and 
http://wjst.wu.ac.th

appropriateness of the length of the program. The answers of the questions were on a 4-level Likert scale ( 4 = strongly agree, $3=$ moderately agree, $2=$ slightly agree, and $1=$ disagree $)$.

Readability is defined as the degree of understandability of written text; in other words, it is an indication of reading ease [36]. In this study, it is referred to the printed health information booklet for mothers. Readability was measured using the Suitability of Assessment of Materials (SAM) questionnaire which was developed by Doak et al. [37]. The questionnaire items checked the suitability of the printed health information materials. The questionnaire covered 6 major evaluation areas, including content, literacy demand, graphics, layout and typography, learning stimulation and motivation, and cultural appropriateness, with a total of 22 items. When answering the questionnaire, there were 3 choices: superior, adequate, and not suitable. Higher SAM scores indicated better suitability of the printed material.

\section{Measurement of maternal participation level}

The maternal participation level was measured by using the Maternal Participation in Caring for a Preterm Infant's Checklist developed by the researcher and consisting of 16 items, with a total score of 16. The content validity of this checklist was approved by the same group of panel experts of the MP Program with the content validity index of 0.94 . The checklist covered 6 care practice activities, with dichotomous answers (yes/no). The score was used to measure the level of maternal participation and provide feedback. If the mother's participation level was less than $95 \%$, the researcher and participants discussed the barriers or obstacles to participating in care practices.

\section{Outcomes of preterm infants}

Outcomes of preterm infants were based on body weight and changes in neurobehavioral function. Body weight. A digital weight scale was used to measure the body weight. The focus was on the amount of increase of the preterm infant's body weight. The digital weight scale was calibrated by the company. It was a digital scale with the standard error analysis of 0.01 kilograms, and a tare function where the scale can be reset to zero. Before each usage, the scale was placed on a straight smooth floor and read "stable" as well as reset to zero; $500 \mathrm{~g}$ standard weight to test the accuracy of the scale.

Changes in neurobehavioral function. The neonatal neurobehavioral examination (NNE) scale, developed by Morgan, Koch, Lee, and Aldag [34], was used to measure changes in neurobehavioral function. The developers of the scale authorized the use of the scale for the study. The scale reflects the different changes in neurobehavioral function as a preterm infant's age increases. The scale consists of 27 items, organized into 3 dimensions: 1) tone and motor patterns, 2) primitive reflexes, and 3) behavioral responses. Each dimension consists of 9 items and is scored on a 3-point Likert scale. The total scores ranged from 27 to 81 . Higher total scores indicated better gestational maturation and neurobehavioral status. Reliability of the Neonatal Neurobehavioral Examination (NNE) Scale was tested by inter-rater methods among 3 preterm infants. Inter-rater method was conducted by the research assessor and physical therapist who was an expert in preterm infant neurodevelopment examination. Both of them independently used the scale to examine the same preterm infants at the same time. The calculation index of agreement of inter-rater observer reliability was 0.96 .

\section{Results and discussion}

The analysis was on the demographic of mothers and preterm infants, and the program, regarding feasibility, acceptability, readability, and preterm infants' outcomes.

Maternal demographic data. Participants of this study were 3 married mothers whose ages were 27, 30 , and 32. In terms of educational level, one mother had completed a diploma, and 2 mothers had completed a bachelor's degree. One mother was a housekeeper, the other 2 included a business owner and a government employee. Their family income ranged from 788 to 2,364 US dollars per month. Two of the mothers delivered by cesarean section, and one by normal labor. The length of hospital stay of 2 mothers was 4 days, and the other was 14 days. 
http://wjst.wu.ac.th

The demographic data of preterm infants found that their genders were 2 boys and one girl. They were born with a gestational age of 28,30 , and 32 weeks, respectively. In terms of birth order within their families, 2 of the preterm infants were the first born, and the other was the second born. Their birth weights were 920,1160 , and $1330 \mathrm{~g}$. The infants' size for gestational age was classified as one of small for gestational age, and 2 of appropriate for gestational age. Their Apgar scores at the first minute were 5, 2 , and 8, and at the first five minutes were 6, 2, and 8. The Clinical Risk Index for Babies (CRIB) scores were 10,3 , and 1 , respectively. All of them were diagnosed with respiratory distress syndrome, with mild, moderate, and severe levels, and were hospitalized in an NICU. They received total parenteral nutrition (TPN), lipids, and breast milk in their medical regimen.

The Maternal Participation Program was used to test feasibility of the program in 3 mothers. Figure 1 shows that 3 mothers performed first time diverse care practices at different day. Each mother visited their preterm infant at least twice a day, and recorded activities that they had done to their preterm infant. It was found that mothers of preterm infants with more weeks of gestational age were able to perform maternal participation activities sooner than the other mothers. In addition, after the completion of the Maternal Participation Program, all 3 mothers showed an increase in the level of maternal participation.

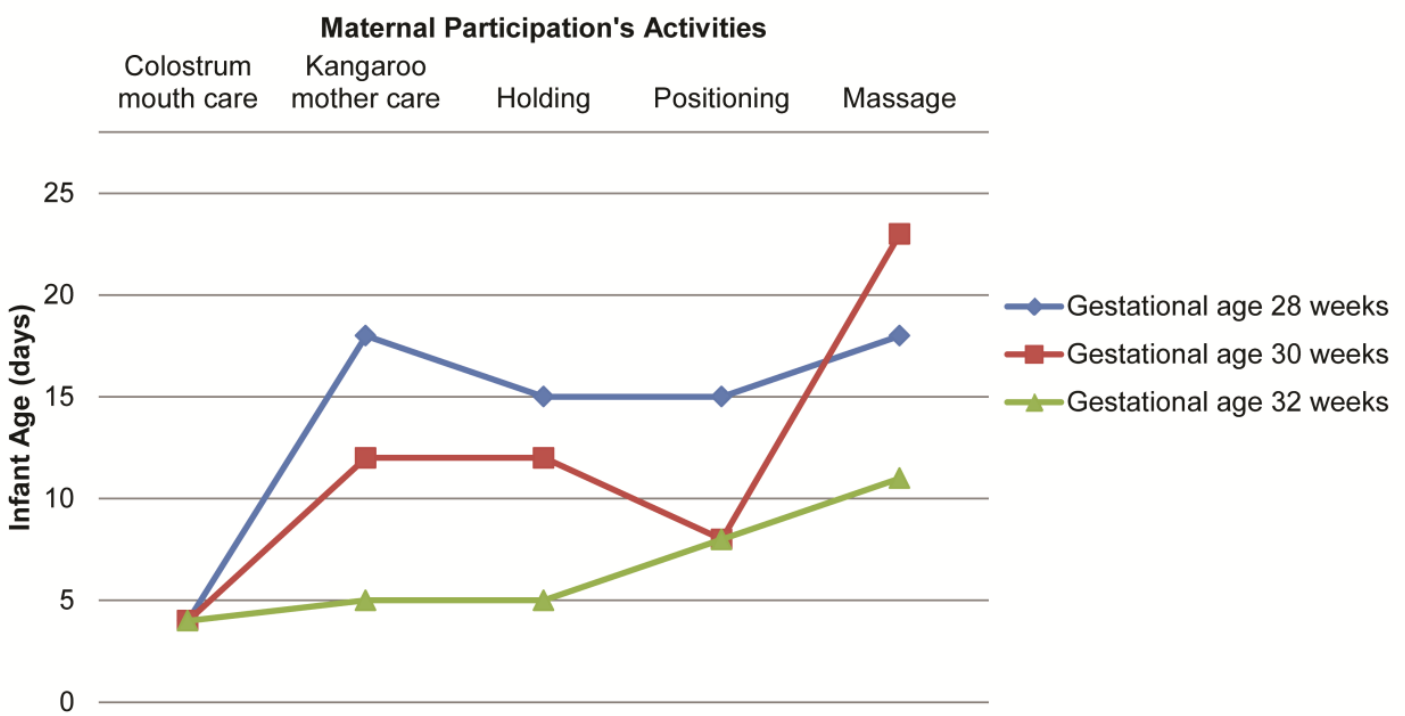

Figure 1 First time diverse care practices activities of maternal participation among 3 mothers.

Maternal participation in caring for preterm infants at day 14. Maternal participation in caring for preterm infants with a gestational age of 28, 30 and 32 weeks showed a performance of 75.0, 87.5, and $93.8 \%$, respectively. During the first two weeks, the mothers of the preterm infants with the older gestational age participated more in caring for their infants. All of them performed activities related to providing a healing environment, safeguarding sleep, and positioning and handling. They spent less time doing kangaroo mother care and massage. The mother with the 32-week gestational age infant performed kangaroo mother care and massage when her preterm infant was 5 days old and 11 days old. None of the mothers performed facilitated tucking or kangaroo mother care for their preterm infant during painful procedures.

Maternal participation in caring for preterm infants at day 21. Maternal participation in caring for preterm infants with a gestational age of 28,30 and 32 weeks increased performance to $93.8,100$, and $100 \%$, respectively. All of the mothers helped to minimize stress and pain for their preterm infants, by handling during procedures of taking blood, giving parenteral medication, and doing secretion suction. Only the mother of the preterm infant of 30 weeks gestation performed kangaroo mother care for her 
http://wjst.wu.ac.th

preterm infant when the infant was receiving a painful procedure at 17 days of life. In approximately the third week, the mothers with the 28 -week gestation and 30 -week gestation infants gave their preterm infants their first massage at age 18 days and 23 days, respectively.

All the mothers agreed that the program is practical and provides a useful guideline for caring for preterm infants. The teaching strategies that were used made all the mothers feel comfortable to ask for further information, helping them learn about their preterm infants. The program provided the mothers with a goal when visiting their infants, encouraged the mothers to form good relationships with the NICU nurses, and enabled them to cope with the NICU environment, thereby reducing stress and increasing their participation in taking care of their infants.

During implementation of the program, the researcher provided assistance, facilitation, and encouragement to mothers to booster the maternal participation level. It was found that facilitation, such as feeding colostrum or fresh milk, doing kangaroo mother care, or doing massage, gave the mothers a chance to participate in the care of their preterm infants during a visit. Information of maternal stress reduction was stated by mother during the acceptability evaluation as "as receiving the information about my baby make my stress reduced". Other strategies to encourage the mothers were telephone contact and chat support (Line application) via social media. Recording the infant's body weight before and after giving a measured amount of expressed breastmilk, as explained in the "My Baby" handbook, helped encourage the 3 mothers/participants to give breast milk exclusively to their infants. In addition, seeing the infants' weight change and knowing the cause of weight loss during the first two weeks helped to reduce the mothers' anxiety and gave them inspiration to give expressed breast milk, which in turn promoted their infants' sleep and improved the infants' weight gain.

The Maternal Participation Program was used to test acceptability of the program in 3 mothers. All 3 mothers commented on the program in terms of the benefits, timing, and method of teaching. They expressed that the Maternal Participation Program was helpful and facilitated learning about infant care by means of explanation and demonstration. They also stated that the program contributed to increased support, served their needs, and helped them gain self-confidence in caring for their preterm infants. In terms of timing, all of them favored a 4-day teaching course beginning the first week after delivery, with one hour of lecture and one hour of practice per session, and one day off to review what they had learned. The 3 participants were impressed with the content of the "multi-touch interactive multimedia" that was developed with high-definition video, clear sound, and easy-to-understand language, and rated it as "most satisfactory". They suggested rearranging the presentation of activities to comply with the preterm infants' clinical manifestations. The 3 participants agreed that practicing with an infant model beforehand made it easier for them to perform maternal participation activities with their preterm infants. They also appreciated the fact that practicing with their preterm infants increased their opportunity to participate in taking care of their infants. They rated this activity as "most satisfactory".

The handbook was used to test readability of the program in three mothers. All participants rated the content, literacy demand, layout and typography, learning stimulation and motivation, and cultural appropriateness at a superior level. They rated the graphics, handbook size, topic of recording, and order of practices at an adequate level. In addition, they suggested that the order of the contents should be the same order as in the multi-touch interactive multimedia.

Preterm infant outcomes were evaluated based on gained body weight and neurobehavioral development at day 14 and day 28 after birth.

According to infant weight gain, comparing birth weight at day 14 and day 28 after birth, it was found that the 3 preterm infants all gained weight. Interestingly, the preterm infants who were gestationally older gained more weight than the younger infant (Table 1). The preterm infants with 28, 30 , and 32 weeks of gestation regained to their birth weight at day 10, day 12, and day 9, respectively (Figure 2). 
http://wjst.wu.ac.th

Table 1 Comparison of weight gain among 3 cases compared to their birthweight.

\begin{tabular}{lccc}
\hline Infant weight & $\begin{array}{c}\text { Case 1 } \\
\mathbf{( 2 8} \text { weeks) }\end{array}$ & $\begin{array}{c}\text { Case 2 } \\
\mathbf{( 3 0} \text { weeks) }\end{array}$ & $\begin{array}{c}\text { Case 3 } \\
(\mathbf{3 2} \text { weeks) }\end{array}$ \\
\hline Birth weight $(\mathrm{gm})$ & 920 & 1160 & 1330 \\
Weight at day 14 after admission $(\mathrm{gm})$ & 950 & 1180 & 1425 \\
Weight at day 28 after admission $(\mathrm{gm})$ & 1210 & 1460 & 1810 \\
Mean weight gain rate at day 14 $(\mathrm{gm} / \mathrm{d})$ & 3.6 & 8.0 & 13.9 \\
Mean weight gain rate at day 28 $(\mathrm{gm} / \mathrm{d})$ & 20.7 & 20.3 & 26.8 \\
\hline
\end{tabular}

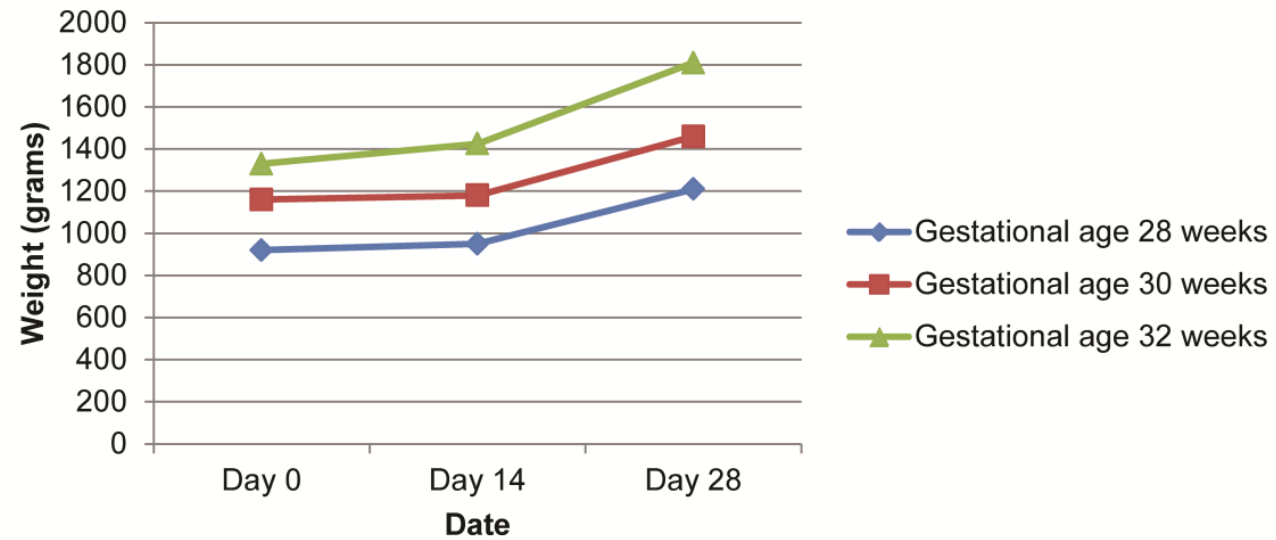

Figure 2 Linear graph showing comparison of weight gain among the 3 preterm infants.

Neurobehavioral development of preterm infants increased their overall neurobehavioral development scores and dimension scores on day 14 and day 28 after birth (Table 2). In terms of the dimension of tone and motor pattern, all of the items had increased scores of arm recoil, but there were no increased scores for popliteal ankle, slip through, pull to sit, and head righting. For primitive reflexes, all of the infants had increased scores on sucking. Positive support, walking, Moro, and tonic neck reflexes showed no increased scores. The preterm infants with a gestational age of 34 weeks and 36 weeks demonstrated the rooting reflex. In terms of the behavioral responses dimension, there were increased scores in orientation, irritability, peak of excitement, and self-quieting, but scores on cuddliness were not increased. 
http://wjst.wu.ac.th

Table 2 Neonatal neurobehavioral examination (NNE) scores at day 14 and day 28 after implementing the program.

\begin{tabular}{rccc}
\hline NNE scores & Case 1 & Case 2 & Case 3 \\
At day 14 after admission & (28 weeks) & (30 weeks) & weeks) \\
Tone and motor patterns & 12 & 14 & 15 \\
Primitive reflexes & 14 & 14 & 15 \\
Behavioral responses & 14 & 14 & 16 \\
\hline Total score & $\mathbf{4 0}$ & $\mathbf{4 2}$ & $\mathbf{4 6}$ \\
\hline At day 28 after admission & 15 & 18 & 17 \\
Tone and motor patterns & 17 & 16 & 22 \\
Primitive reflexes & 20 & 21 & $\mathbf{5 7}$ \\
\hline Behavioral responses & $\mathbf{5 2}$ & $\mathbf{5 5}$ & \\
\hline
\end{tabular}

\section{Conclusions}

The results of these 3 case studies provide additional information about education and monitoring strategies to promote maternal participation in caring for preterm infants and positive infant health outcomes using an individual approach. The procedures of the program, as evaluated by the 3 mothers, were suitable in terms of content, method, and duration. In addition, the implementation of monitoring strategies, consisting of evaluation of the maternal participation level, as well as assistance, facilitation, and encouragement for boosting maternal participation, resulted in increased, regular, and ongoing maternal participation.

All 6 care practices helped to increase the mean weight gain velocity of the 3 preterm infants at the age of 2 weeks, which was similar to another study of weight gain velocity among very preterm infants after birth [33]. In addition, they all regained their birthweight within 2 weeks. Furthermore, the 3 preterm infants showed increased neurobehavioral development scores at day 28 after birth. Throughout the program, there was no harm done to any of the preterm infants as a result of maternal participation.

All mothers pointed out that the implementation of the Maternal Participation Program based on the Neonatal Integrative Developmental Care model was appropriate for mothers in an NICU and Thai context. The program also helped them to overcome the obstacles caused by their "kreng jai" culture and fear of doing harm to their preterm infants. The program was implemented within the first two days after delivery, which is the golden period of bonding with a premature infant. The program helped the mothers to become familiar with an NICU environment, along with the 6 care practices which were considered to be the best practices of neuroprotective interventions [24]. It also changed the culture of care among nurses in the NICU to more fully support maternal participation. The outcomes of the program for mothers and preterm infants were achieved as expected. However, this positive feedback came from only 3 mothers in this pilot study.

There should be a repetition of the program with a revision of educational strategies based on feedback from the participating mothers. Educational strategies should provide one day off between each session for the mother to review and practice what she has learned. Multimedia should be produced in the form of 2-way communication with the VDO illustrating real time preterm infants' conditions. It may motivate mothers to actively participate in the caring of their preterm infants. The education and monitoring strategies in the components of the Maternal Participation Program for enhancing growth and neurobehavioral development of preterm infants need to be carried out in a randomized controlled trial design to assure the effectiveness of the program. 
http://wjst.wu.ac.th

\section{Acknowledgements}

The authors would like to express their deepest appreciation to the NICU staff at Maharaj Nakorn Chiang Mai Hospital, Thailand for their support, and to the mothers of the preterm infants for their efforts and invaluable assistance to accomplish the aims of the program.

\section{References}

[1] CP Hornik, AL Sherwood, CM Cotten, MM Laughon, RH Clark and PB Smith. Daily mortality of infants born at less than 30 weeks' gestation. Early Hum. Dev. 2016; 96, $27-30$.

[2] S Chawanpaiboon and S Kanokpongsakdi. Preterm birth at Siriraj Hospital: A 9 year period review (2002-2010). Siriraj Med. J. 2011; 63, 143-6.

[3] JB Gouyon, S Iacobelli, C Ferdynus and F Bonsante. Neonatal problems of late and moderate preterm infants. Semin. Fetal Neonatal Med. 2012; 17, 146-52.

[4] RE Behrman and AS Butler. Preterm birth: Causes, Consequences, and Prevention. The National Academies Press, Washington DC, 2007.

[5] MC Sullivan, ME Msall and RJ Miller. 17-year outcome of preterm infants with diverse neonatal morbidities: Part 1-Impact on physical, neurological, and psychological health status. J. Spec. Pediatr. Nurs. 2012; 17, 226-41.

[6] S Jenga, KT Yaub and R Tengb. Neurobehavioral development at term in very low birthweight infants and normal term infants in Taiwan. Early Hum. Dev. 1998, 51, 235-45.

[7] S Cömert, T Ağzıkuru, Y Akin, B Telatar, PD Tan, SG Ergen and P Dervişoğlu. The cost analysis of preterm infants from a NICU of a State Hospital in Istanbul. Iran J. Pediatr. 2012, 22, 185-90.

[8] H Niela-Vilén, A Axelin, S Salanterä, L Lehtonen, O Tammela, R Salmelin and R Latva. Early physical contact between a mother and her NICU-infant in two university hospitals in Finland. Midwifery 2013; 92, 1321-30.

[9] AV Zani, ARC Golias, STF Martins, CMGL Parada, SS Marcon and VLP Tonete. Feelings experienced by the family of an at-risk newborn infant: Integrative literature review. J. Nurs. UFPE On Line 2013; 7, 269-78.

[10] J Malakouti, M Jabraeeli, S Valizadeh and J Babapour. Mothers' experience of having a preterm infant in the Neonatal Intensive Care Unit: A phenomenological study. Iran J. Crit. Care Nurs. 2013; 5, 172-81.

[11] EC Hall, H Kronborg, H Aagaard and BS Brinchmann. The journey towards motherhood after a very preterm birth: Mothers' experiences in hospital and after home-coming. J. Neonatal Nurs. 2013; 19, 109-13.

[12] GC Lasiuk, T Comeau and C Newburn-Cook. Unexpected: An interpretive description of parental traumas' associated with preterm birth. BMC Pregnancy Childbirth 2013; 13, S13.

[13] V Swanson, H Nicol, R McInnes, H Cheyne, H Mactier and E Callander. Developing maternal selfefficacy for feeding preterm Babies in the neonatal unit. Qual. Health Res. 2012; 22, 1369-82.

[14] H Kearvell and J Grant. Getting connected: How nurses can support mother/infant attachment in the neonatal intensive care unit. Aust. J. Adv. Nurs. 2010; 27, 75-82.

[15] R Flacking, L Lehtonen, G Thomson, A Axelin, S Ahlqvist, VH Moran, U Ewald and F Dykes. Closeness and separation in neonatal intensive care. Acta Paediatr. 2012; 101, 1032-7.

[16] EJ Starr-Phillips and AK Beery. Natural variation in maternal care shapes adult social behavior in rats. Dev. Psychobiol. 2013; 56, 1017-26.

[17] KO Brien, M Bracht, K Macdonell, TM Bride, K Robson, LO Leary, K Christie, M Galarza, T Dicky, A Levin and SK Lee. A pilot study of family integrated care in a Canadian neonatal intensive care unit. BMC Pregnancy Childbirth 2013; 13, S12.

[18] MG Welch, MM Myers, PG Grieve, JR Isler, WP Fifer, R Sahni, MA Hofer, J Austin, RJ Ludwig, RI Stark and FNI Trial Group. Electroencephalographic activity of preterm infants is increased by family nurture intervention: A randomized controlled trial in the NICU. J. Clin. Neurophysiol. 2014; 125, 675-84. 
http://wjst.wu.ac.th

[19] P Sannino, ML Giannì, GD Bon, C Fontana, O Picciolini, L Plevani, M Fumagalli, D Consonni and F Mosca. Support to mothers of premature babies using NIDCAP method: A non-randomized controlled trial. Early Hum. Dev. 2016; 95, 15-20.

[20] KM Benzies, JE Magill-Evans, KA Hayden and M Ballantyne. Key components of early intervention programs for preterm infants and their parents: A systematic review and meta-analysis. BMC Pregnancy Childbirth 2013; 13, 1-15.

[21] C Brecht, RJ Shaw, SM Horwitz and NH John. Effectiveness of therapeutic and behavioral interventions for parents of low-birth weight premature infants: A review. Infant Ment. Health J. 2012; 36, 651-65.

[22] J Brett, S Staniszewska, M Newburn, N Jones and L Taylor. A systematic mapping review of effective interventions for communicating with, supporting and providing information to parents of preterm infants. BMJ 2011; 1, e000023.

[23] JA Vanderveen, D Bassler, CM Robertson and H Kirpalani. Early interventions involving parents to improve neurodevelopmental outcomes of premature infants: A meta-analysis. J. Perinatol. 2009; 29; 343-51.

[24] L Altimier and RM Phillips. The Neonatal integrative developmental care model: Seven neuroprotective core measures for family-centered developmental care. Newborn Infant Nurs. Rev. 2013; 13, 9-22.

[25] Y Pongjaturavit and RC Harrigan. Parent participation in the care of hospitalized child in Thai and western cultures. Issues Compr. Pediatr. Nurs. 2003. 26; 183-99.

[26] P Pholanun, S Kantawang and P Klunklin. Maternal participation in caring for preterm infants in neonatal intensive care unit and related factors. Nurs. J. 2013; 40, 89-101.

[27] B Punthmatharith, U Buddharat and T Kamlangdee. Needs, need responses, and need response satisfaction of mothers having infants admitted to a Neonatal Intensive Care Unit of a Regional Hospital in lower southern Thailand. Songkla Med. J. 2007; 5, 117-26.

[28] N Taya, W Picheansatian and R Yunaka. Needs and received response among mothers of newborns in Neonatal Intensive Care Units. J. Health Sci. 2007; 16, 268-74.

[29] R Orapiriya, V Jirapaet and B Rodcumdee. Struggling to get connected: The process of maternal attachment to the preterm infant in the neonatal intensive care unit. Thai J. Nurs. Res. 2007; 11, 251-63.

[30] JH Chaudhuri, MA Easterbrooks and CR Davis. The relation between emotional availability and parenting style: Cultural and economic factors in a diverse sample of young mothers. Parent Sci. Pract. 2009; 9, 277-99.

[31] R Flacking, L Lehtonen, G Thomson, A Axelin, S Ahlqvist, VH Moran, U Ewald and F Dykes. Closeness and separation in neonatal intensive care. Acta Paediatr. 2012; 101, 1032-7.

[32] AL Patel, JL Engstrom, PP Meier, E Robert and RE Kimura. Accuracy of methods for calculating postnatal growth velocity for extremely low birth weight infants. Pediatrics 2005; 116, 466-73.

[33] TR Fenton, R Nasser, M Eliasziw, JH Kim, D Bilan and R Sauve. Validating the weight gain of preterm infants between the reference growth curve of the fetus and the term infant. BMC Pediatr. $2013 ; \mathbf{1 3}, 92$.

[34] AM Morgan, V Koch, V Lee and J Aldag. Neonatal Neurobehavioral examination: A new instrument for quantitative analysis of neonatal neurological status. Phys. Ther. 1988; 68, 1352-8.

[35] E Proctor, H Silmere, R Raghavan, P Hovmand, G Aarons, A Bunger, R Griffey and M Hensley. Outcomes for implementation research: Conceptual distinctions, measurement challenges, and research agenda. Admin. Pol. Ment. Health 2011; 38, 65-76.

[36] BK Redman. The Practice of Patient Education: A Case Study Approach. $10^{\text {th }}$ eds. Mosby Elsevier, St Louis, 2007.

[37] CC Doak, LG Doak and JH Root. Teaching Patients with Low Literacy Skills. $2^{\text {nd }}$ eds. J.B. Lippincott, Philadelphia, 1996. 